\title{
Recomendación de usuarios en una plataforma colaborativa accesible
}

Recibido: 15 Febrero 2014 - Revisado: 30 Abril 2014

Aceptado: 30 Mayo 2014 - Publicado: 30 Julio 2014

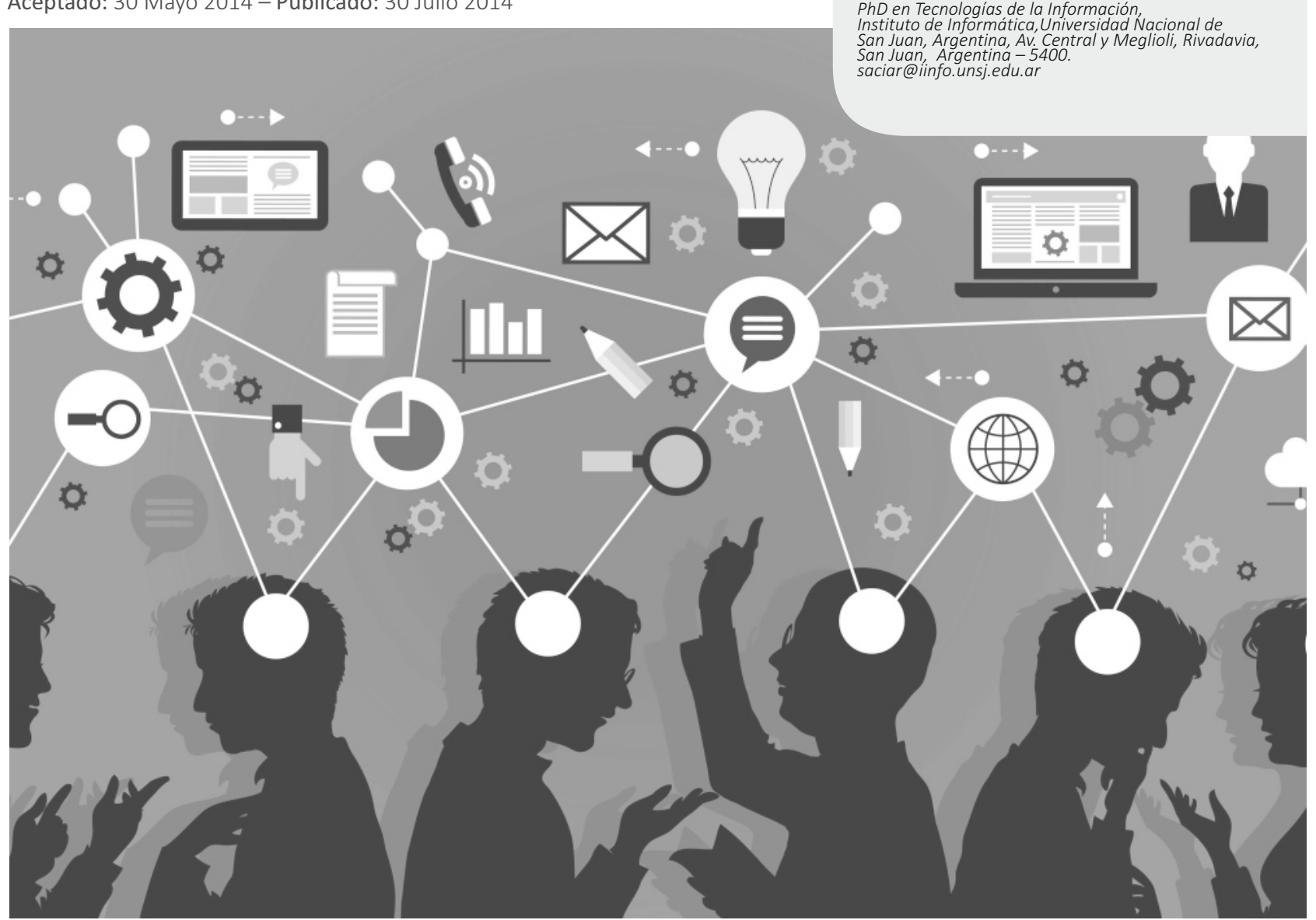

Resumen: El uso de plataformas colaborativas en educación se ha incrementado en los últimos años. La gran cantidad de usuarios registrados en las mismas ha originado el problema de no saber con quién interactuar. Muchas veces la persona con quien se interactúa no es la idónea y resultan interacciones no exitosas. En este artículo, se presenta un sistema recomendado que sugiere personas idóneas para interactuar en una plataforma colaborativa.

Palabras clave: Sistemas recomendadores, personalización, plataformas colaborativas, modelos de usuarios.

Abstract: The use of collaborative platforms in education has increased in recent years. The large number of registered users originated the problem of not knowing who interact. Many times, the person with whom they interact is not ideal, resulting in not successful interactions.

This article presents a system that recommends suitable people to interact in a collaborative platform. Key words: Recommender systems; personalization; collaborative platform; user model. 


\section{INTRODUCCIÓN}

Las Tecnologías de la Información y las Comunicaciones (TIC) desempeñan un rol esencial en la sociedad actual, sobre todo en los procesos educativos. Estas han propiciado el surgimiento del Elearning, que ha facilitado el aprendizaje a través de Internet y la comunicación entre diferentes individuos que físicamente pueden estar en lugares diferentes.

Muchas de las plataformas de E-learning utilizan tecnologías de la Web 2.0, tales como blogs, fórums, chats, wikis, entornos para compartir recursos, plataformas colaborativas que establecen interacciones entre usuarios, etc. Esto permite construir conocimiento en forma colectiva mediante aportaciones colaborativas entre individuos, grupos e instituciones interesados en temáticas comunes. Con la Web 2.0, se involucra directamente al usuario en situaciones donde se articula el conocimiento en torno a la conexión de nodos humanos (Fumero et al., 2007).

Dichos servicios posibilitan a los usuarios interactuar, expresarse y comunicarse a través de espacios colaborativos, específicamente llamados "Plataformas Colaborativas". Estas otorgan la posibilidad que docentes y alumnos puedan entablar relaciones con otros y así, facilita la coordinación, el contacto entre ellos, la colaboración, el compartir materiales y la creación de productos digitales. Además, proporcionan herramientas con un alto grado de accesibilidad e interactividad, donde los usuarios participan en función de sus intereses, situación académica, lugar de trabajo, región geográfica, entre otras.

Pero, no es tan fácil hacer uso de tales plataformas, especialmente cuando son usuarios que no se conocen y están distribuidos físicamente. Es aquí donde se presenta como solución otra herramienta de la Web 2.0: Ios Sistemas Recomendadores. Un sistema recomendador sugerirá a un usuario de una plataforma colaborativa otro usuario con quien interactuar (Aciar y Aciar, 2013).

En este trabajo, se presenta una propuesta de un Sistema Recomendador de Usuarios con base en su perfil y reputación en una plataforma colaborativa.

\section{SISTEMAS RECOMENDADORES}

Regularmente estamos expuestos a una cantidad de información que se extiende de una forma más rápida que nuestra capacidad para comprenderla. Los Sistemas Recomendadores surgen para facilitar la toma de decisiones en temas\dominios en los que las opciones son numerosas y variadas.

Estos sistemas son utilizados para indicar a los usuarios que los visitan, información, productos y otros ítems; tratan de automatizar aspectos de un modelo de búsqueda de información completamente distinto al tradicional donde, por ejemplo, los usuarios reciben información a partir de las elecciones realizadas por otras personas con gustos similares (Langs y Santos, 2006). Un Sistema Recomendador ayuda al usuario inexperto a elegir a través de las opciones que va a seleccionar; lo impulsa a la adquisición de la mejor opción definición que Resnick y Varian (1997) ha dado a los sistemas de recomendación.

Cabe destacar que en la investigación efectuada para el presente trabajo final, se encontraron varios ejemplos de Sistemas de Recomendación. Uno de los más distinguidos es la librería Amazon.com, que dispone de uno de los recomendadores colaborativos más conocidos y usados en el ambiente del comercio electrónico. En este sistema recomendador cada producto adquirido y valorado por un usuario lo empareja con productos análogos, y después combina aquellos productos parecidos en una lista de recomendación. 
Tenemos, entonces, que habitualmente en un Sistema Recomendador, se contrasta el perfil del usuario con algunas características de referencia de los ítems (estas características pueden basarse en la relación de similitud del usuario con el ítem, utilizando información de otros usuarios con preferencias o intereses similares a él) y busca predecir el ranking o valoración que el usuario le daría a un ítem que aún el sistema no ha considerado.

Para crear un perfil de un usuario con sus gustos, preferencias e intereses, se puede utilizar dos formas o métodos en la recolección de información: implícita o explícita (Mingus, 2013). La información implícita: Es aquella que el sistema adquiere a partir del comportamiento e interacción del usuario con el sistema. Por ejemplo: registro de los ítems que el usuario ha visto en una tienda online, analizar el número de visitas que recibe un ítem; analizar las redes sociales de las que el usuario forma parte $y$, de esta manera, conocer sus gustos y preferencias; hacer un análisis del historial de compra del usuario; comparar el tiempo que el usuario pasa leyendo sobre ciertos ítems en el sitio en contraste contra el tiempo que dedica a otros ítems; introducción de palabras clave en una búsqueda; analizar la cantidad de clics que hace el usuario sobre determinada sección del sitio. La información explícita: Es la información que el usuario ingresa al sistema en respuesta a las peticiones manifiestas del mismo. Por ejemplo, solicitar al usuario que pondere un conjunto de ítems de una lista de ítems, presentar al usuario dos ítems, y solicitarle que seleccione uno de ellos, solicitar al usuario que cree una lista de ítems de su preferencia, formulario de registración al sistema, cuestionario con preguntas sobre sus preferencias, intereses, etc.

En la literatura existen varios métodos para realizar las recomendaciones; estos son conocidos como filtrados. A continuación se enumeran: Filtrado Colaborativo: Es una de las técnicas más empleadas por los recomendadores colaborativos. El término filtrado colaborativo se ha comenzado a utilizar en las últimas décadas, pero el concepto es algo que las personas han utilizado desde siempre: compartir opiniones entre sí, de boca en boca; de esta manera se filtra la información con base en las opiniones de nuestros conocidos. Hay al menos dos tipos de filtrado colaborativo, uno basado en el usuario (user - based) y el otro basado en los productos (ítems-based). Filtrado por contenido: En este filtrado las sugerencias de los ítems se basan en deducciones sobre las necesidades de los usuarios y sus preferencias. Es decir, se utiliza conocimiento en donde se tiene la información sobre cómo un ítem específico responde a una necesidad particular del usuario y, por lo tanto, la razón sobre la relación entre la necesidad y una posible recomendación. Filtrado Demográfico: Clasifican a los usuarios de acuerdo a su perfil y hacen las recomendaciones basándose en clases demográficas, es decir, las variables que se tienen en cuenta, tales como la edad, el sexo, el lugar de origen, etc., permitirán ubicar a un usuario en uno u otro grupo (Recomendador, 2013). Filtrado Híbrido: Estos recomendadores surgen como combinación de otros enfoques (por ejemplo, basados en contenido y colaborativos). Son, sin lugar a dudas, los Sistemas Recomendadores más complejos de construir debido a que combinan múltiples técnicas en un único sistema y consiguen una participación activa de todas ellas.

\section{PERFIL DEL USUARIO}

Las creencias y los deseos de un usuario se obtienen por medio del modelado de usuario; a través de éste se pueden saber los intereses sobre ciertos recursos y qué recursos derivan otros intereses para un usuario. Un modelo de usuario es, entonces, una representación explícita de las propiedades de un usuario específico. Se utiliza para razonar acerca de las necesidades, preferencias o comportamiento futuro del usuario (Gaudioso, 2002) (Pazzani y Billsus, 2001). 


\section{Perfil diversidad-Accesibilidad}

El perfil diversidad dentro de la plataforma colaborativa contiene información acerca de las condiciones y preferencias de acceso a la información de los docentes registrados. El perfil contempla características tecnológicas como de conducta, comportamiento y físicas de los docentes, que se deben tener en cuenta al momento de brindar una mejor accesibilidad en la interacción dentro de la plataforma. Estas características pueden ser: capacidad de Reconocimiento de emociones, capacidad para manejar la conducta, trastorno por déficit de atención e hiperactividad (TDAH), nivel de audición, manejo de ayudas auditiva, forma de comunicación, tipo de limitación visual, manejo de ayudas visuales, limitación motora, manejo de herramientas tecnológicas para limitación motora y preferencias de acceso.

\section{RECOMENDADOR DE USUARIOS EN BASE AL PERFIL Y REPUTACIÓN}

Debido a las nuevas posibilidades educativas hoy en día, los docentes y estudiantes se verán envueltos en nuevas formas de trabajar y relacionarse entre ellos. Estas nuevas formas involucran herramientas TICS que optimizan los procesos comunicativos y permiten la reutilización de información y conocimiento. La mayor de las ventajas del uso de las TICs es la posibilidad de interacción rompiendo las barreras del tiempo y el espacio, donde por ejemplo profesores de diferentes establecimientos educativos pueden trabajar en el armado de un curso, e incluso en el dictado del mismo sin necesidad de ser en el mismo lugar físico o en el mismo momento. Como lo mencionamos anteriormente las plataformas colaborativas ofrecen la posibilidad de interactuar distribuidos físicamente en diferentes lugares del mundo. Una de las posibles interacciones entre docentes es la de interactuar para planificar un curso para las personas con necesidades diversas (problemas visuales, auditivos, sociales, etc).
Problema: Un docente ingresa a la plataforma colaborativa para comenzar a interactuar con otro docente sobre alguna temática, este docente es la primera vez que ingresa a la plataforma y no conoce qué otros usuarios están registrados en dicha plataforma. Se estima que la plataforma sea utilizada por muchos docentes de las diversas universidades participantes de esta red, por lo que resulta en un gran número de docentes que podrían estar registrados en ella.

Solución: el sistema recomendará otro usuario (Usr - Usuario recomendado) para iniciar la interacción.

La recomendación será realizada en base a la similitud del perfil $(P)$ del usuario y el valor de reputación (R) que es obtenido del resultado de interacciones pasadas de ese usuario dentro de la plataforma.

$$
U_{u s r}=\operatorname{Recomendador}(P, R)
$$

El recomendador realizará como primera instancia un proceso de comparación entre los perfiles de los usuarios. Una vez finalizado el mismo, y de haber desestimado varios usuarios que no presentan similitudes de perfiles, el sistema comenzará a efectuar un proceso para determinar la confianza entre usuarios. De esta manera, puede efectuar la recomendación al usuario que ha solicitado la interacción para la planificación del curso.

A continuación, se describirán los dos parámetros que son utilizados en las recomendaciones.

\section{Perfil del Usuario Docente (P)}

El perfil del docente será utilizado por el recomendador para obtener información sobre el conocimiento, formación y experiencia de los usuarios. Esta información puede ser proporcionada directamente por él de manera explícita, por ejemplo, mediante un formulario. A continuación, se describen 
dos tipos de información que debe contener el perfil del usuario:

Datos demográficos: Son aquellos datos inherentes a los usuarios, los que van con ellos, y solo a ellos les corresponden: nombre y apellido; dirección, teléfono fax y correo electrónico; fecha de nacimiento; nacionalidad.

Datos Profesionales: Los datos profesionales permiten determinar de un usuario su experiencia y conocimiento: Títulos académicos; puestos docentes desempeñados; actividad docente desempeñada: en centros universitarios, en centros no universitarios; actividad investigadora: disciplina de conocimiento, grupo actual de investigación, temas de investigación, colaboración con otros grupos de investigación; nivel de competencia: avanzado, intermedio, inicial; publicaciones: libros y capítulos en libros, libros publicados, libros editados, capítulos de libros; publicaciones artículos en revistas: catalogadas en el JCR del ISI, otras revistas publicaciones en actas de congresos. Idiomas.

Perfil diversidad: capacidad de Reconocimiento de emociones, capacidad para manejar la conducta, trastorno por déficit de atención e hiperactividad (TDAH), nivel de audición, manejo de ayudas auditiva, forma de comunicación, tipo de limitación visual, manejo de ayudas visuales, limitación motora, manejo de herramientas tecnológicas para limitación motora y preferencias de acceso.

La información obtenida del perfil nos permite obtener información acerca del conocimiento, experiencia y situación académica actual de los usuarios. En este caso particular planteado en este trabajo se ha realizado una equivalencia convirtiendo al currículum vitae de los docentes por perfiles, el cual será adquirido de forma explícita y manual por el docente.

\subsection{Reputación (R)}

La reputación puede derivarse de la confianza que tienen ciertos miembros de una comunidad hacia uno en particular. La confianza depende de contextos y situaciones particulares de acción: no se tiene confianza siempre y en toda circunstancia. Para Barón (1988), la virtud de la confianza es la tendencia a comportarse como si uno creyera que otros se comportarán de manera correcta y la tendencia a valorar dicho comportamiento en uno mismo y en otros. Una virtud es un tipo de norma social que aceptamos en otros y por implicación en nosotros mismos.

La reputación requiere de una fundamentación que se sitúa por encima de las obligaciones morales que surgen de la esfera familiar y por debajo de las regulaciones legales que provienen de la esfera institucional. De acuerdo con la teoría de la elección racional, la emergencia de la reputación en los sistemas sociales se sostiene en la estructura de incentivos de largo plazo que las relaciones económicas involucran.

La reputación de un usuario es la opinión que los otros pares tienen acerca de él. La misma es un valor sobre una escala cualitativa o cuantitativa calculada para cada usuario.

Es importante destacar que existen diferentes mecanismos de reputación, estos se han aplicado en diversos campos tales como el comercio electrónico (Noriega et. al, 1998), los sistemas de recomendación (Montaner et.al, 2002). En el presente trabajo final, el valor de reputación $\mathrm{R}$ es obtenido a partir de las interacciones pasadas realizadas entre los usuarios (docentes).

La reputación de un docente es la opinión que los otros docentes tienen acerca de él; esta opinión está basada en el registro de las interacciones positivas e interacciones negativas ejecutadas por ellos. 
La medida de reputación que se utilizará es la especificada por Jigar Patel (Patel et.al., 2005). Ellos definen el valor de reputación en un intervalo entre $(0,1)$, en el cual 0 significa un usuario poco confiable y 1, un usuario confiable. Este valor es obtenido usando la siguiente ecuación:

$$
T(S)=E[B s=\alpha ; \beta]_{(2)}
$$

Donde E se calcula de la siguiente manera:

$$
E[B s=\alpha ; \beta]_{(2)}=\frac{\alpha}{\alpha+\beta}
$$

Los parámetros $\alpha$ y $\beta$ están definidos como:

$$
\begin{aligned}
& \alpha=m_{s}+1 \\
& \beta=n s_{+1}
\end{aligned}
$$

Donde $\alpha$ es el número de interacciones positivas +1 y $\beta$ es el número de interacciones negativas +1 .

Una interacción positiva es aquella en la cual un docente haya valorado exitosamente una interacción con otro docente. Tenemos, por ejemplo, un docente $A$ ha terminado de interactuar con un docente $B$, el sistema les pide que valores su interacción en positiva o negativa.

Proceso de Selección del Docente para Recomendar en base al Perfil (P) y Reputación ( $R$ )

Por ejemplo el docente A desea crear un curso conjunto de matemática y solicita que se le recomiende otro docente con el que pueda interaccionar. El sistema recomendador selecciona el docente con el que va a interactuar de acuerdo al perfil y la reputación del mismo y se lo presenta al docente.
Para este proceso se cuenta con:

Un usuario solicitante us es el usuario que ingresa al sistema y pide recomendación de usuarios para interactuar.

Un conjunto de usuarios candidatos cuc $=\{u c 1, u c 2$, uc3,....ucn\} son los usuarios que están registrados en la plataforma.

Un usuario recomendado ur que es el usuario seleccionado por el sistema del conjunto de usuarios candidatos (cuc) para presentarlo al usuario solicitante (us)

Teniendo en cuenta esta información, el proceso para recomendar se realiza de la siguiente forma:

\section{Registro del Usuario}

Al ingresar al sistema un usuario us que desea interactuar con otro docente el sistema verifica si es un usuario registrado, si es un usuario registrado recupera su perfil, caso contrario le pide que se registre al sistema. La registración conlleva a la petición de información sobre su perfil.

\section{Recuperación y comparación de perfiles similares}

Una vez que se crea el perfil de usuario, el sistema recomendador recupera los usuarios con perfiles similares, estos no necesariamente tienen que ser iguales. Así, crea un conjunto de usuarios candidatos a ser recomendados cuc $=\{u c 1, u c 2, u c 3, \ldots . u c n\}$.

Para comparar los perfiles se utiliza el modelo Booleano basado en la lógica de proposiciones de Boole. Mediante este modelo, los perfiles son representados por vectores, donde cada elemento del vector es función si un criterio del perfil a ser comparado está presente o no en el perfil a ser comparado. 
Por ejemplo, los criterios a ser tenidos en cuenta para la comparación de los perfiles son el idioma, la disciplina de conocimiento y diversidad.

Si el usuario us tiene en su perfil idioma=español, disciplina de conocimiento=Lenguaje $y$ diversidad=disminución auditiva.

Obtener el valor de reputación de los usuarios candidatos

Una vez que se obtiene el conjunto de usuarios candidatos a ser recomendados $\mathrm{cuc}=\{\mathrm{uc} 1, \mathrm{uc} 2$, uc3,....ucn $\}$; para cada uno de ellos, se obtiene el valor de reputación $\mathrm{R}$ aplicando la ecuación № 2 .

Las interacciones positivas o negativas resultan de la valoración que otorga un usuario después de interactuar con otro. La primera vez que ingresa un usuario al sistema, el valor de $\mathrm{R}$ es 0,5 , ya que no posee valoración de otros usuarios.

El sistema se basará en aquellos que contengan un perfil idéntico al docente que solicita que se le recomiende otro usuario. Lo primero es el proceso de comparación de perfiles, en donde los parámetros que se utilizarán para dicha comparación serán el idioma, disciplina de conocimiento y diversidad.

\section{EVALUACIÓN}

Para la evaluación de la propuesta, se utilizó un conjunto de datos provenientes de las interacciones realizadas por docentes que usaron un prototipo de la plataforma colaborativa. El conjunto de datos contiene la siguiente información:

Información de las interacciones entre pares de usuarios desde noviembre de 2011 hasta junio de 2012.
Información de 50 usuarios, profesores y estudiantes a profesores en las universidades de los países del Mercosur.

Información del perfil demográfico y académico de esas personas.

Valoración de los usuarios participantes de las interacciones dadas por los mismos usuarios intervinientes. Esta valoración es binaria: Buena o Mala.

Se realizaron experimentos utilizando el recomendador de usuarios con base en el perfil y la reputación de los usuarios.

Se realizaron experimentos recomendando usuarios para interactuar aleatoriamente.

Se dividió el conjunto de datos en dos: un conjunto de datos para las pruebas con interacciones desde noviembre de 2011 hasta marzo de 2012. Y otro conjunto de testeo con las interacciones desde abril de 2012 hasta junio de 2012. Este último conjunto es el que se utiliza para obtener los usuarios que volvieron a interactuar con los mismos usuarios.

Para la comparación de los perfiles se tuvieron en cuenta los criterios de idioma, disciplina de conocimiento y diversidad.

Se utilizó el método booleano para la comparación de los perfiles representados como vectores booleanos. Se utilizó la ecuación (2) para la obtención de la reputación de los usuarios.

Se realizaron 500 recomendaciones de usuarios con base en el perfil y la reputación y 500 recomendaciones de usuarios en forma aleatoria.

Las evaluaciones de las recomendaciones se realizaron midiendo la cantidad de interacciones que 
fueron calificadas como buenas por los usuarios, bajo el supuesto que interacciones con usuarios recomendados en forma precisas resultan en interacciones positivas buenas. Los Gráficos. 1 y 2 muestran las calificaciones de las interacciones de usuarios recomendados con base en el perfil y reputación y calificaciones de interacciones de usuarios recomendados de forma aleatoria, respectivamente.

Como se observa en los gráficos, la calificación de las interacciones de los usuarios recomendados con base en el perfil y la reputación es mayor la cantidad de positivas (1) que negativas (-1). Esto quiere decir que la mayoría de los usuarios a los cuales se les recomendó utilizando la propuesta realizada en este trabajo estuvieron satisfechos con las interacciones con sus pares.

Caso contrario, las interacciones realizadas con usuarios recomendados en forma aleatoria resultaron mayormente insatisfactorias (-1).

\section{CONCLUSIONES}

Como conclusión, se puede decir que los aportes del trabajo son: una herramienta para plataformas colaborativas que permite sugerir usuarios para interactuar de forma personalizada; un nuevo aporte a los métodos de recomendación con base en el conocimiento al tener en cuenta el perfil y la reputación para realizar las recomendaciones.

El sistema recomendador presentado en este trabajo se convierte en una contribución importante para las plataformas colaborativas. Los nuevos usuarios de una plataforma colaborativa no saben quién es la persona más idónea para comenzar a interactuar sobre alguna temática, muchas veces las interacciones no son exitosas debido a que la persona con quien interactuó resulta no ser la idónea. El sistema recomendador propuesto es una solución a este problema, le recomienda a una persona un usuario adecuado con quien interactuar.

Para demostrar la validez de este sistema recomendador, se realizaron experimentos cuyos resultados demuestran que usuarios que interactuaron con usuarios recomendados de forma personalizada (con base en su perfil y la reputación del mismo dentro la plataforma colaborativa) fueron más exitosas.

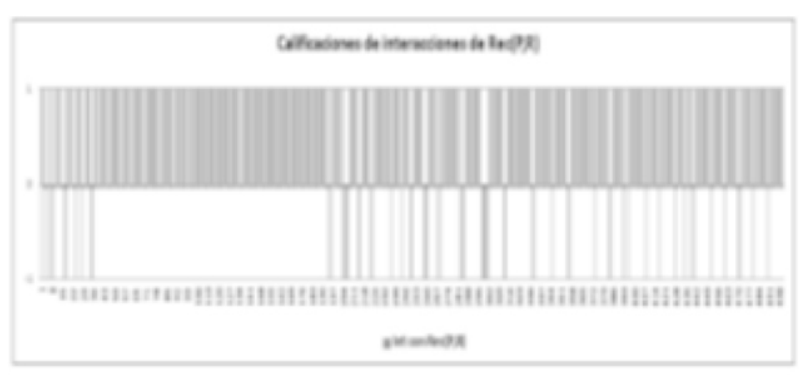

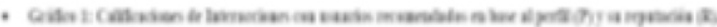

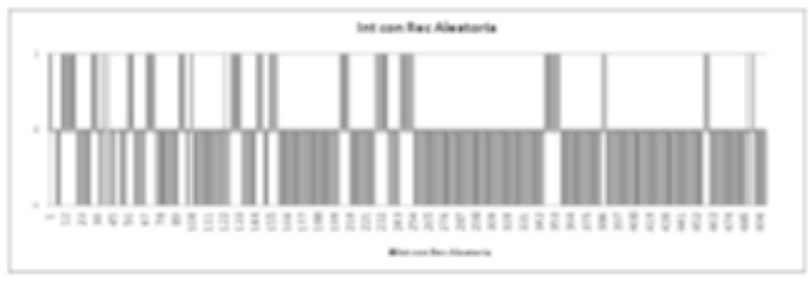

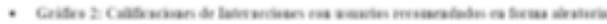




\section{REFERENCIAS}

Aciar, G. y Aciar, S (2013). Recomendador de usuarios en una plataforma colaborativa en base a su perfil y reputación. 42 JAIIO- ASAI 2013. Córdoba, Argentina. $1-8$

Any Other Day (2010). Recomendadores. Recuperado de:

http://anyotherday.wordpress.com/2008/12/03/pla neando-un-recomendador-para-sistemas-e-learning. Baron, R. A. (1988). Atributions and Organizational Confict: The mediating role of apparent Sincerity. Organizational Behavior and Human ecision processes, 41, 111-127.

Fumero, Roca y Sáez (2007). Web 2.0 [en línea]. Fundación Orange, 8-65. Disponible en Www.fundacionauna.com/areas/25_publicaciones/ WEB_DEF_COMPLETO.pdf.

Gaudioso, E. (2002). Contribuciones al Modelado del Usuario en Entornos Adaptativos de Aprendizaje y Colaboración a través de Internet mediante técnicas de Aprendizaje Automático (Tesis de doctorado). Universidad Nacional de Educación a Distancia., Bogotá.

Mingus, C. (2013). Charles Mingus. Recuperado de: http://es.wikipedia.org/wiki/Charles_Mingus.

Montaner, M., López, B., y De la Rosa, J. L. (2002). Opinion-based filtering through trust. Gerona: Institut d'Informa'tica i Aplicacions.

Noriega, P., Sierra, C. y Rodríguez, J.A (1998). The Fishmarket Project. Reflections on Agent-mediated institutions for trustworthy ECommerce. Position Paper at the Workshop on Agent Mediated Electronic Commerce (AMEC-98; Seoul).

Patel, J., Teacy, W. T. L., Jennings, N. R. y Luck, M. (2005). A Probabilistic Trust Model for Handling
Inaccurate Reputation Sources. In Proceedings of Third International Conference on Trust Management (in press).

Pazzani, W. y Billsus, D. (2001). Machine Learning for User Modeling. En Kluwer Academic Publishers (Eds), User Modeling and User-Adapted Interaction 11 (pp. 19-29). Berlín: Kluwer Academic Publishers.

Resnick, P. y Varian, H. R. (marzo, 1997). Recommender systems. Commun. ACM, 40(3), 56-58. Vélez Langs, O. Santos, C. (2006). Aproximando a los Sistemas Recomendadores desde los Algoritmos Genéticos. Revista Colombiana de Computación, 8(2), 7-2 